\title{
Lavado de manos: prevención de infecciones nosocomiales en una clínica de podología
}

\author{
Handwashing: prevention of nosocomial infections at a podiatry clinic \\ Victoria Candelario Poteleschenko*, Jose Luis Muñoz Sánchez ${ }^{* *}$, \\ Javier Navarro Marruedo ${ }^{* * *}$, José María Blanco Rodriguez ${ }^{* * * *}$ \\ * Ingeniero técnico en Informática y estudiante de podología \\ vcandela@estumail.ucm.es \\ ** Técnico especialista en laboratorio y diagnóstico clínico y estudiante de podología \\ joseguis16@hotmail.com \\ *** Estudiante de podología \\ javinavarro46@gmail.com \\ **** Profesor Titular de Universidad. Dr. por la Universidad Complutense de Madrid. Enfermero \\ jmblanco@enf.ucm.es
}

\author{
Correspondencia \\ Victoria Candelario Poteleschenko \\ Calle del Mezquite 2, Portal B, esc 2, $6^{\circ} \mathrm{B}$ \\ 28045 Madrid (España) \\ E-mail:vcandela@estumail.ucm.es
}

Fecha de recepción: 20 julio de 2012

Fecha de aceptación: 15 de noviembre de 2012

Los autores declaran no tener ningún tipo de interés económico o comercial.

\section{RESUMEN}

La transferencia de microorganismos a través de las manos del personal sanitario ha sido identificada como el factor más importante en la transmisión de infecciones en medios hospitalarios. Ministros de salud y funcionarios superiores, expertos técnicos y la Organización Mundial de la Salud (OMS) sugieren una serie de medidas fundamentales para luchar contra las infecciones asociadas a la atención sanitaria, también conocidas como infecciones nosocomiales, que ponen en peligro los avances obtenidos con grandes esfuerzos en los ámbitos de la salud y la esperanza de vida. Una de las medidas primordiales para evitar estas infecciones, es el lavado de manos. Debido a su repercusión y la importancia de este tema se realiza una encuesta en una Clínica Universitaria de Podología, para identificar los conocimientos del personal que trabaja o hace practicas en esta clínica sobre la prevención de este tipo de infecciones, así como para conocer la frecuencia con la que realizan el lavado de manos y si se realiza de manera correcta.

Palabras clave: lavado de manos; infecciones nosocomiales; prevención; higiene.

\begin{abstract}
The transfer of microorganisms via the hands of medical staff has been identified as the most important factor in the transmission of infections in hospitals or Health Clinics. Health ministers, technical experts and the World Health Organization (WHO) suggest some measures to combat hospital - acquired infections, also known as nosocomial infections. One of the main measures to avoid these infections is hand washing. For the importance of this topic an inquiry was conducted in a university clinic of podiatry, to identify knowledge of the people working there, or doing practices on prevention of these infections, and to determine the frequency with which they perform hand washing and if they performed correctly.
\end{abstract}

Key words: wash hands; nosocomial infections; prevention; hygiene.

Sumario: 1. Introducción, 2. Objetivos generales, 3. Objetivos específicos, 4. Material y métodos, 5. Resultados, 6. Discusión, 7. Conclusiones, Bibliografía.

Referencia normalizada: Candelario Poteleschenko, V., Muñoz Sánchez, JL., Navaro Marruedo, J., Blanco Rodríguez, JM. Lavado de manos: prevención de infecciones nosocomiales en una clínica de podología. Rev. Int. Cienc. Podol. 2013; 7(1): 49-55. 


\section{INTRODUCCION}

Una infección nosocomial puede definirse de la manera siguiente: una infección contraída en el hospital por un paciente internado por una razón distinta de esa infección. Una infección que se presenta en un paciente internado en un hospital o en otro establecimiento de atención de salud en quien la infección no se había manifestado ni estaba en período de incubación en el momento del internado. Comprende las infecciones contraídas en el hospital, pero manifiestas después del alta hospitalaria y también las infecciones ocupacionales del personal del establecimiento ${ }^{1}$.

La transferencia de microorganismos a través de las manos del personal ha sido identificada como el factor más importante en la transmisión de infecciones.

Según una nueva publicación de la Iniciativa Mundial en pro de la Seguridad del Paciente, «Una atención limpia es una atención más segura», en todo momento hay en el mundo más de 1,4 millones de personas gravemente enfermas a consecuencia de esas infecciones. Entre el 5\% y el $10 \%$ de los pacientes que son ingresados en hospitales en los países desarrollados contraen estas infecciones, según el informe. En algunos países en desarrollo, el porcentaje de pacientes afectados puede superar el $25 \%{ }^{2}$.

Por su propia naturaleza, las infecciones relacionadas con la atención sanitaria tienen una etiología multifacética, relacionada con los sistemas y procesos de prestación y atención sanitaria y las limitaciones políticas y económicas de los sistemas de salud y los países, así como el comportamiento humano condicionado por la educación ${ }^{3}$.

La higiene de las manos es la medida primordial para prevenir dichas infecciones. Aunque se trata de una acción sencilla su incumplimiento entre los dispensadores de acción sanitaria representa un problema en todo el mundo ${ }^{3}$.

La mayor parte de las defunciones y sufrimiento causados por las infecciones relacionadas con la atención sanitaria pueden evitarse. Existen ya prácticas baratas y sencillas para prevenirlas. La higiene de las manos, una acción muy simple, sigue siendo la medida primordial para reducir su incidencia y la propagación de los microorganismos resistentes a los antimicrobianos, lo que mejora la seguridad del paciente en todos los ámbitos. Sin embargo, el cumplimiento de las normas de higiene de manos en todo el mundo es muy escaso, por lo que los gobiernos deberían velar por que el fomento de dicha higiene reciba la atención y los fondos suficientes para que resulte eficaz ${ }^{3}$.

Hace años que se dispone de medidas para prevenir las infecciones relacionadas con la infección sanitaria. Lamentablemente, por diversas razones no se han aplicado; una de ellas es la deficiente formación y observancia en materia de prácticas de higiene de las manos de eficacia demostrada ${ }^{3}$.

Una acción sencilla, como aparentemente es la higiene de manos, está condicionada por una serie de factores que la hacen compleja, estos factores son básicamente; momentos para la higiene, producto utilizado, accesibilidad a puntos de higiene, tiempo empleado, técnica adecuada, uso de guantes, usos de joyas y formación ${ }^{4}$.

\section{OBJETIVOS GENERALES}

Identificar los conocimientos, que sobre el lavado de manos y la prevención de infecciones nosocomiales, tienen los podólogos de más de tres años de experiencia, podólogos profesionales con menos de dos años de experiencia, personal de asistencia sanitaria, y alumnos en prácticas que prestan servicio en la Clínica de Podología en la que se realiza la encuesta.

\section{OBJETIVOS ESPECIFICOS}

1. Conocer la frecuencia con la que el personal de una Clínica de Podología se lava las manos en la actividad diaria.

2. Identificar los conocimientos que tiene el personal de una Clínica de Podología sobre infecciones nosocomiales.

3. Identificar los conocimientos que tiene el personal de una Clínica de Podología sobre la importancia de la prevención de infecciones en medios sanitarios a partir de una buena higiene de manos. 


\section{MATERIAL Y METODOS}

Se realiza una encuesta de doce preguntas sobre el conocimiento básico de infecciones nosocomiales, uso de los guantes e higiene de manos en el medio sanitario, así como un cuestionario para conocer la frecuencia con la que el personal de la Clínica se lava las manos en un día de trabajo normal. Se realiza a los podólogos de más de tres años de experiencia, podólogos profesionales con menos de dos años de experiencia, personal de asistencia sanitaria, y alumnos en prácticas.

Se procede a validar la encuesta antes de llevarla a cabo en la Clínica para comprobar si se ajusta a los objetivos que se pretenden conseguir, así como si se entienden correctamente las preguntas planteadas en la misma.

Se procede a entregar la encuesta individualmente y de manera confidencial a la muestra de estudio, se les da un tiempo prudente para responder a las preguntas y se pasa a recoger.

MUESTRA (número de personal). Podólogos con más de tres años de experiencia: 6 , personal de servicios: 13 , podólogos profesionales con menos de dos años de experiencia: 13, número de alumnos en prácticas: 116 .

\section{RESULTADOS}

Con relacion a los resultados obtenidos en el lavado de manos atendiendo a los años del ejercicio profesional, hemos encontrado que los podologos con una experiencia de mas de tres años son los que mejor conocen el protocolo, mientras que los que tienen menos experiencia no lo conocen tan bien como se observa en la Figura 1. En cuanto al personal de servicios de la clínica según la encuesta son los que menos conocimientos demostraron sobre el lavado de manos como se observa en la Figura 2. Los alumnos que se encuentran realizando practicas en la Clínica a pesar de tener menos experiencia han demostrado conocer bien el protoclo sobre el lavado de manos como se muestra en la Figura 3. Se aprecia que los podologos con menos de dos años de experiencia conocen bien el protocolo como se observa en la Figura 4. Respecto a la frecuencia con la que el personal de la clínica en la que se llevo la encuesta se lava las manos se observa que una parte importante del personal se lava siempre las manos al pasar de una zona contaminada del paciente a una zona limpia, aunque un alto porcentaje solo lo realiza a veces como se ob-

\section{RESPUESTAS CORRECTAS DE LOS PODOLOGOS PROFESIONALES CON MAS DE TRES AÑOS DE EXPERIENCIA ( $\mathrm{N}=3)$}

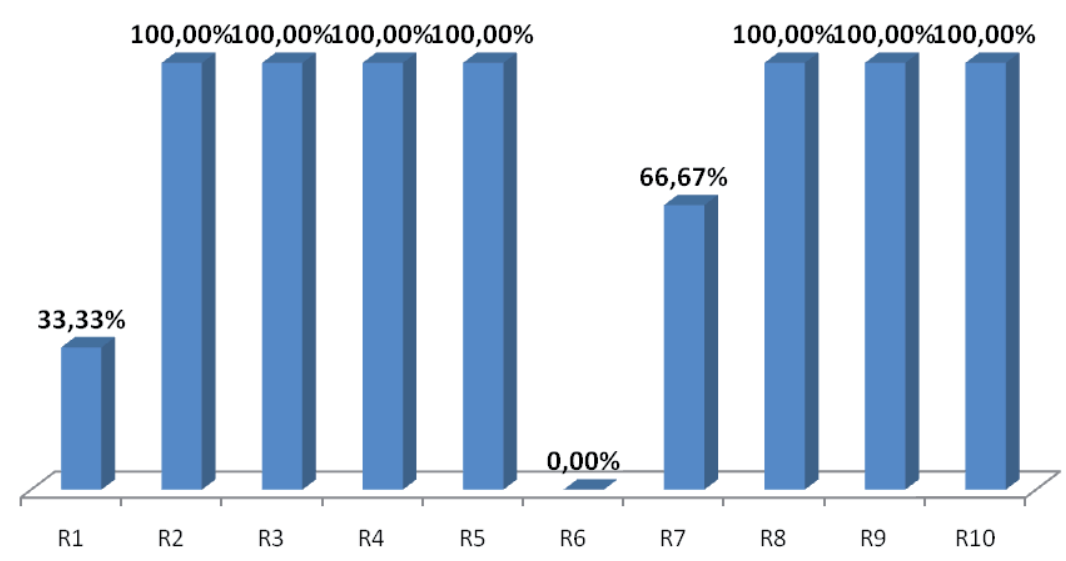

Figura 1. En el gráfico se observa que los podólogos profesionales con más de tres años de experiencia tienen muy buenos conocimientos respecto al lavado de manos, casi en todas las preguntas el 100\% contestó correctamente. 


\section{RESPUESTAS CORRECTAS DE LOS PAS (PERSONAL DE SERVICIOS)}

$(\mathrm{N}=8)$

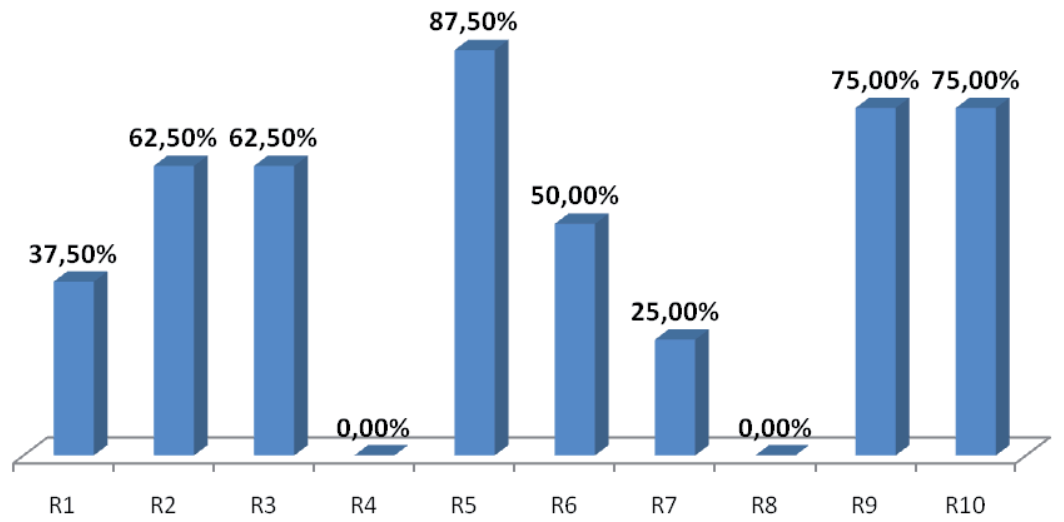

Figura 2. En el gráfico se pone de manifiesto que existe un buen conocimiento sobre el lavado de manos entre el personal de servicios. En 6 de 10 preguntas más del 50\% contestó correctamente y en la pregunta 5: Dentro de las medidas de prevención y control de infecciones, usted considera que el lavado de manos es; R5: Es la base principal para la prevención y control de infecciones en medios sanitarios; una de las preguntas más importantes de la encuesta, casi el 100\% contestó correctamente.

\section{RESPUESTAS CORRECTAS DE LOS ALUMNOS PRÁCTICAS \\ $(\mathrm{N}=78)$}

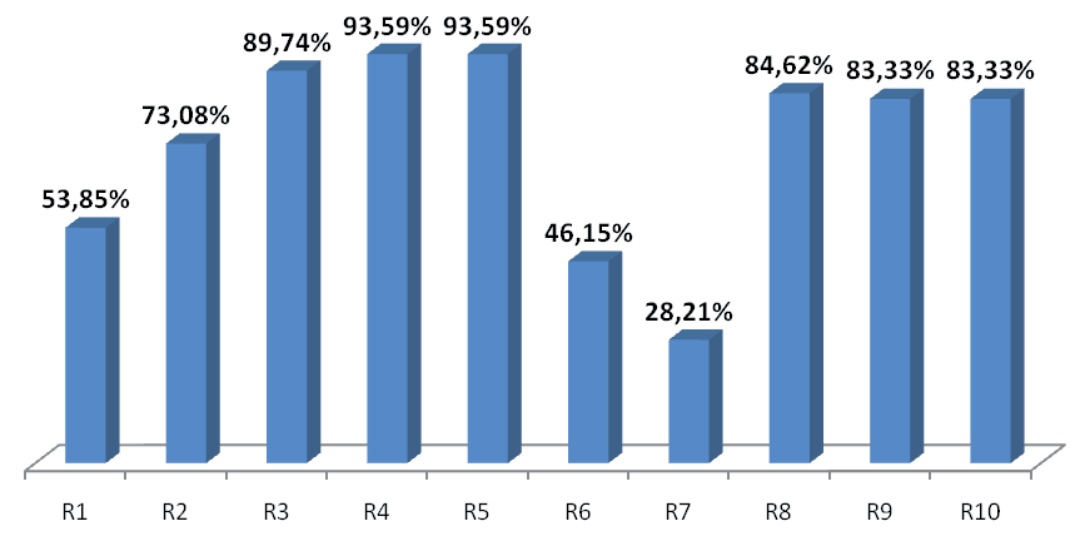

Figura 3. En el gráfico se muestran las preguntas contestadas correctamente por los alumnos que se encuentran realizando prácticas en la clínica. Como se puede observar los conocimientos respecto al lavado de manos son muy buenos, aproximadamente el 100\% contestó correctamente a las preguntas de la encuesta. 
serva en la Figura 5. Se puede comprobar que a pesar de conocer bien el protocolo existen algunos puntos que necesitan reforzarse y crear conciencia entre el personal, ya que algunos no se lavan las manos nunca o solo lo hacen a veces antes del contacto directo con el paciente, o antes de ponerse los guantes como se observa en la Figura 6.

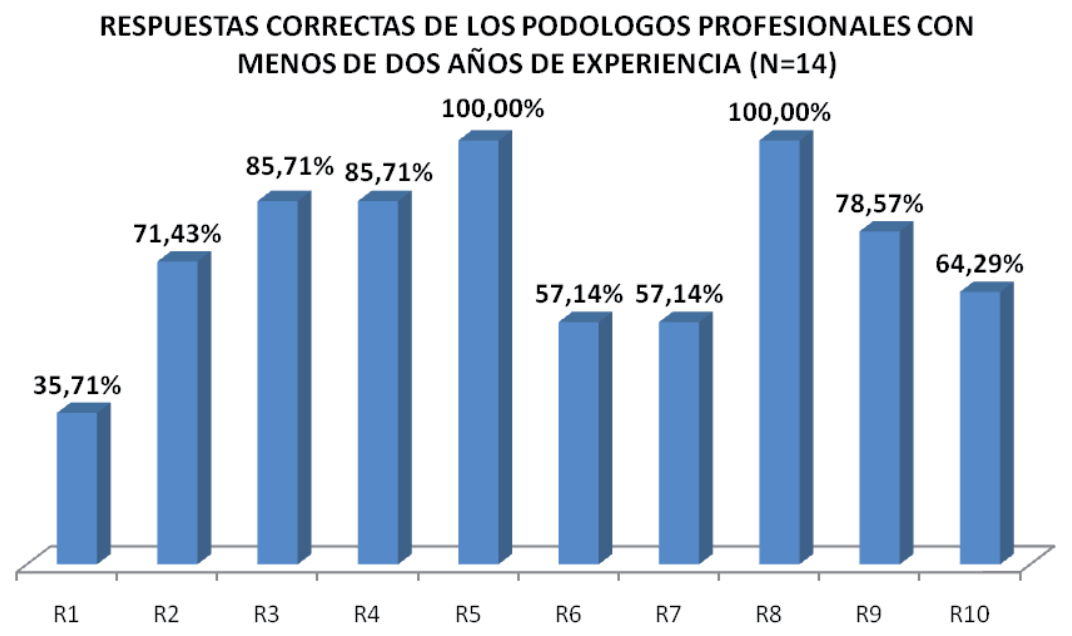

Figura 4. El grafico muestras las respuestas contestadas correctamente por los podólogos profesionales con menos de dos años de experiencia. Se observa que existe un buen conocimiento respecto al lavado de manos, en casi todas las preguntas cerca del $100 \%$ contestó correctamente.

\section{¿AL ATENDER AL PACIENTE, CUANDO SE PASE DE UN AREA DEL CUERPO CONTAMINADA A OTRA LIMPIA? $(\mathrm{N}=102)$}

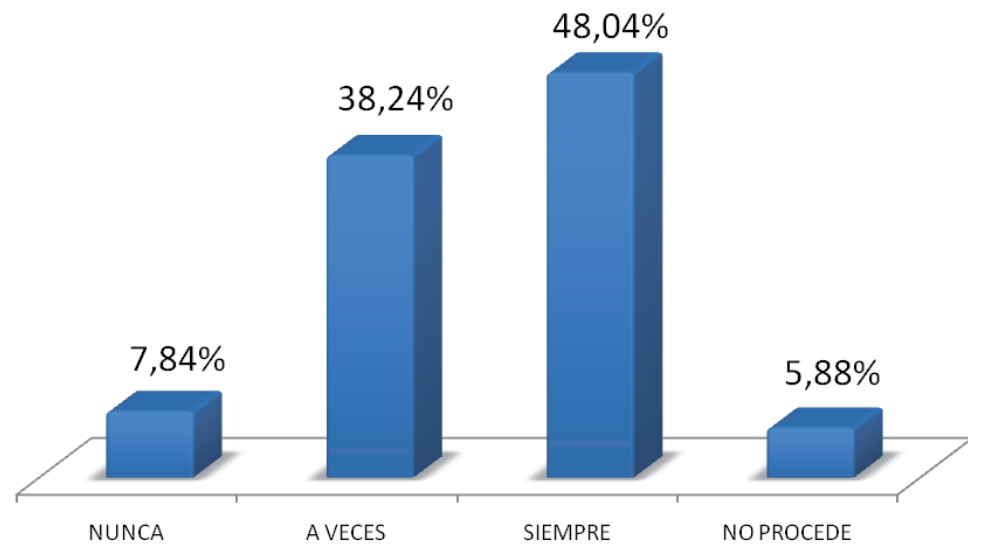

Figura 5. En el gráfico se muestra la frecuencia con la que el personal de la Clínica encuestada se lava las manos en una jornada de trabajo habitual al atender un paciente y pasar de una zona contaminada a una zona limpia. Se aprecia que existe una buena adherencia al lavado de manos ya que casi un $50 \%$ se las lava a veces o siempre. 


\begin{tabular}{|c|c|c|c|c|}
\hline Te lavas las manos... & Nunca & A veces & Siempre & No procede \\
\hline ¿Al iniciar la jornada? & 1 & 41 & 60 & 0 \\
\hline ¿Al finalizar la jornada? & 1 & 14 & 87 & 0 \\
\hline ¿Antes del contacto directo con pacientes? & 2 & 41 & 59 & 0 \\
\hline $\begin{array}{l}\text { ¿Después de entrar en contacto con líquidos } \\
\text { o excreciones corporales, mucosas, piel no } \\
\text { intacta o vendajes de heridas? }\end{array}$ & 0 & 17 & 85 & 0 \\
\hline $\begin{array}{l}\text { ¿Después de entrar en contacto con objetos } \\
\text { inanimados en la inmediata vecindad del } \\
\text { paciente? }\end{array}$ & 8 & 39 & 49 & 6 \\
\hline $\begin{array}{l}\text { ¿Al atender al paciente, cuando se pase } \\
\text { de un área del cuerpo contaminada a otra } \\
\text { limpia? }\end{array}$ & 11 & 42 & 46 & 3 \\
\hline $\begin{array}{l}\text { ¿Antes y después de manipular } \\
\text { medicamentos? }\end{array}$ & 11 & 44 & 40 & 7 \\
\hline ¿Tras la utilización de los servicios WC? & 0 & 3 & 99 & 0 \\
\hline $\begin{array}{l}\text { ¿Antes y después de la manipulación de } \\
\text { cualquier sistema de drenaje? }\end{array}$ & 1 & 4 & 87 & 10 \\
\hline $\begin{array}{l}\text { ¿Antes y después de la utilización de } \\
\text { guantes? }\end{array}$ & 0 & 55 & 47 & 0 \\
\hline
\end{tabular}

Figura 6. En la tabla se muestra una serie de preguntas realizadas al personal de la Clínica para conocer la frecuencia con la que realizaban el lavado de manos en situaciones habituales en una jornada laboral. Se observa que existe una buena adherencia al lavado de manos.

\section{DISCUSION}

Según los resultados de la encuesta se observa que en general existen buenos conocimientos sobre el lavado de manos en la clínica de podologia seleccionada para este trabajo, podemos apreciar que la experiencia en el ejercicio de la profesión influye positivamente en cuanto a los conocimientos y aplicación del protocolo del lavado dde manos para prevenir las infecciones nosocomiales aunque según la encuesta a pesar de conocer los protocolos no se aplican correctamente en todo momento como se observa en la figura 6 , ya que algunos profesionales no se lavan las manos nunca por ejemplo al atender al paciente, o al pasar de una zona contaminada a otra limpia. Es posible que los horarios de trabajo, la falta de personal y la necesidad de no tener muchos pacientes en espera contribuyan a que no se cumpla correctamente el protocolo pero es totalmente necesario crear conciencia entre los profesionales de la necesidad de llevar a cabo una buena higiene de manos en todo momento en el que se atiende a un paciente, sobretodo si son pacientes diabeticos con ulceras abiertas con riesgo de infeccion.

\section{CONCLUSIONES}

1. Se evidencia en general que los conocimientos sobre la importancia del lavado de manos para la prevención de infecciones nosocomiales del personal de la Clínica de Podología en la que se ha llevado a cabo la encuesta son buenos.

2. Se evidencia que existe una buena adherencia al lavado de manos. 
3. Se evidencia que el uso de guantes supone una barrera para la correcta higiene de manos.

4. A partir de los resultados obtenidos se tra- baja en base a los puntos necesarios para mejorar la adherencia al lavado de manos por el personal de la clínica de podologia en la que se llevo a cabo la encuesta.

\section{BIBLIOGRAFIA}

1. Prevención de las infecciones nosocomiales. Guía práctica; $2^{\circ}$ edición [consultado 23 feb 2012; 20:30H]. Disponible en: http://www.who.int/csr/resources/publications/drugresist/en/PISpanish3.pdf.

2. Página oficial de la OMS (Organización Mundial de la Salud) [Consultado el 23 feb del 2012; 20:23H] http://www.who.int/mediacentre/news/releases/2005/pr50/es/index.html.

3. Organización mundial de la salud. Directrices de la OMS sobre higiene de manos en la Atención Sanitaria (Borrador avanzado): Resumen. En: Unas manos limpias son manos mas seguras. Ginebra: OMS; 2005. http://www.who.int/patientsafety/information_centre/Spanish_HH_Guidelines.pdf (consultado el 23 de febrero de 2012; 15:00H).

4. Evaluación de la técnica de higiene de manos en profesionales asistenciales. Departamento de Enfermería, Hospital de Terrassa, Consorci Sanitari de Terrassa, España; C.Ramon-Canton*, N. BoadaSanmartin y L. Pagespetit-Casas. 